\title{
DATA NEEDS FOR HYPERSPECTRAL DETECTION OF ALGAL DIVERSITY ACROSS THE GLOBE
}

By Heidi Dierssen, Astrid Bracher, Vittorio Brando, Hubert Loisel, and Kevin Ruddick

A group of 38 experts specializing in hyperspectral remote-sensing methods for aquatic ecosystems attended an interactive Euromarine Foresight Workshop at the Flanders Marine Institute (VLIZ) in Ostend, Belgium, June 4-6, 2019. The objective of this workshop was to develop recommendations for comprehensive, efficient, and effective laboratory and field programs to supply data for development of algorithms and validation of hyperspectral satellite imagery for micro-, macro- and endosymbiotic algal characterization across the globe. The international group of researchers from Europe, Asia, Australia, and North and South America (see online Supplementary Materials) tackled how to develop global databases that merge hyperspectral optics and phytoplankton group composition to support the next generation of hyperspectral satellites for assessing biodiversity in the ocean and in food webs and for detecting water quality issues such as harmful algal blooms. Through stimulating discussions in breakout groups, the team formulated a host of diverse programmatic recommendations on topics such as how to better integrate optics into phytoplankton monitoring programs; approaches to validating phytoplankton composition with ocean color measurements and satellite imagery; new database specifications that match optical data with phytoplank- ton composition data; requirements for new instrumentation that can be implemented on floats, moorings, drones, and other platforms; and the development of international task forces.

Because in situ observations of phytoplankton biogeography and abundance are scarce, and many vast oceanic regions are too remote to be routinely monitored, satellite observations are required to fully comprehend the diversity of micro-, macro-, and endosymbiotic algae and any variability due to climate change. Ocean color remote sensing that provides regular synoptic monitoring of aquatic ecosystems is an excellent tool for assessing biodiversity and abundance of phytoplankton and algae in aquatic ecosystems. However, neither the spatial, temporal, nor spectral resolution of the current ocean color missions are sufficient to characterize phytoplankton community composition adequately. The near-daily overpasses from ocean color satellites are useful for detecting the presence of blooms, but the spatial resolution is often too coarse to assess the patchy distribution of blooms, and the multiband spectral resolution is generally insufficient to identify different types of phytoplankton from each other, even if progress has undeniably been achieved during the last two decades (e.g., IOCGG, 2014). Moreover, the methods developed for multichannel sensor use are often highly tuned to a region but are inaccu- rate when applied broadly.

New orbital imaging spectrometers are being developed that cover the full visible and near-infrared spectrum with a large number of narrow bands dubbed "hyperspectral" (e.g., TROPOMI, PRISMA, EnMAP, PACE, CHIME, SBG). Hyperspectral methods have been explored for many years to assess phytoplankton groups and map seafloor habitats. However, the utility of hyperspectral imaging still needs to be demonstrated across diverse aquatic regimes. Aquatic applications of hyperspectral imagery have been limited by both the technology and the ability to validate products. Some of the past hyperspectral space-based sensors have suffered from calibration artifacts, low sensitivity in aquatic ecosystems (e.g., CHRIS, HICO), and very low spatial resolution (e.g., SCIAMACHY), but the next generation of sensors are planned to have high signal-to-noise ratio and improved performance over aquatic targets. Providing data to develop and validate hyperspectral approaches to characterize phytoplankton groups across the globe poses new challenges. Several recent studies have documented gaps that need to be filled in order to assess algal diversity across the globe (IOCCG, 2014; Mouw et al., 2015; Bracher et al., 2017), which promoted/inspired the formation of this workshop. 


\section{WHAT PHYTOPLANKTON \\ METRICS CAN BE LINKED TO HYPERSPECTRAL IMAGERY?}

The workshop was initially targeted differentiating algal blooms, but the participants felt that the broader field of characterizing phytoplankton groups (PGs) should be considered. The term PG refers to a clustering of species (irrespective of taxonomic affiliation) that can be optically differentiated using remotesensing methods (Bracher et al., 2017). As defined here, PGs do not necessarily have to serve different ecological or biogeochemical functional roles (IOCCG, 2014). Furthermore, PGs based on taxonomic criteria can be referred to as phytoplankton types (PTs), and PGs based on their size range can be referred to as phytoplankton size classes.

With hyperspectral data, several researchers have independently demonstrated with field and laboratory data that mixtures of major PTs (e.g., diatoms, Prochlorococcus [cyanobacteria], coccolithophores) can be differentiated for members contributing largely total chlorophyll $a$ (Bracher et al., 2009; Xi et al., 2015; Organelli et al., 2017; Catlett et al., 2018). Multispectral imagery can only capture the average trends in the open ocean related to dominant PGs (Figure 1). An understanding of the bloom mixtures can be applied to assessing ecosystem diversity, ecological processes, water quality in terms of ecosystem diversity, and food quality, and it should open up new hyperspectral imagery research and applications. For example, cryptophytes may not be the dominant phytoplankton in surface waters, but their fractional presence (e.g., 20\% of PGs) may indicate a healthy ecosystem due to their high food quality in the trophic web.

Differentiation of algal blooms, in particular, first requires defining what constitutes an algal bloom for remote-sensing purposes. Ecologists often define a bloom based on a statistical increase in phytoplankton biomass above an average baseline, and the term is relative to each region (Carstensen et al., 2015; Friedland et al., 2018). For example, the $90^{\text {th }}$ percentile chlorophyll $a$ concentration can be calculated from long-term satellite data archives on a pixel-by-pixel basis to give a threshold for definition of an "algal bloom" (Park et al., 2010). Alternate metrics could be developed based on specified thresholds of chlorophyll $a$ or other indices that include optical properties.

Various taxa-specific algorithms have been developed for diverse aquatic ecosystems; however, such algorithms are not globally applicable. They have not been demonstrated to be unique to the specific taxa and can be based on pigment-specific features that span different phytoplankton groups. Differentiating dinoflagellates and diatoms globally may be extremely challenging because they exhibit similar spectral absorption and large intraspecies variability (Organelli et al., 2017; Catlett and Siegel, 2018). Hence, a user cannot simply apply these approaches to identify specific taxa widely across different aquatic ecosystems. Such extrapolation will lead to high uncertainty and confusion, with users not knowing when and where a specific product is viable. More global data sets are needed to address such problems.

Because most phytoplankton groups are highly variable in size, shape, and cellular levels of pigment, algorithms for estimating pigment concentration are usually associated with large uncertainty when applied globally. Workshop presentations showed various methods for assessing pigment composition using hyperspectral reflectance, including Gaussian deconvolution, differential optical absorption spectroscopy, princi-
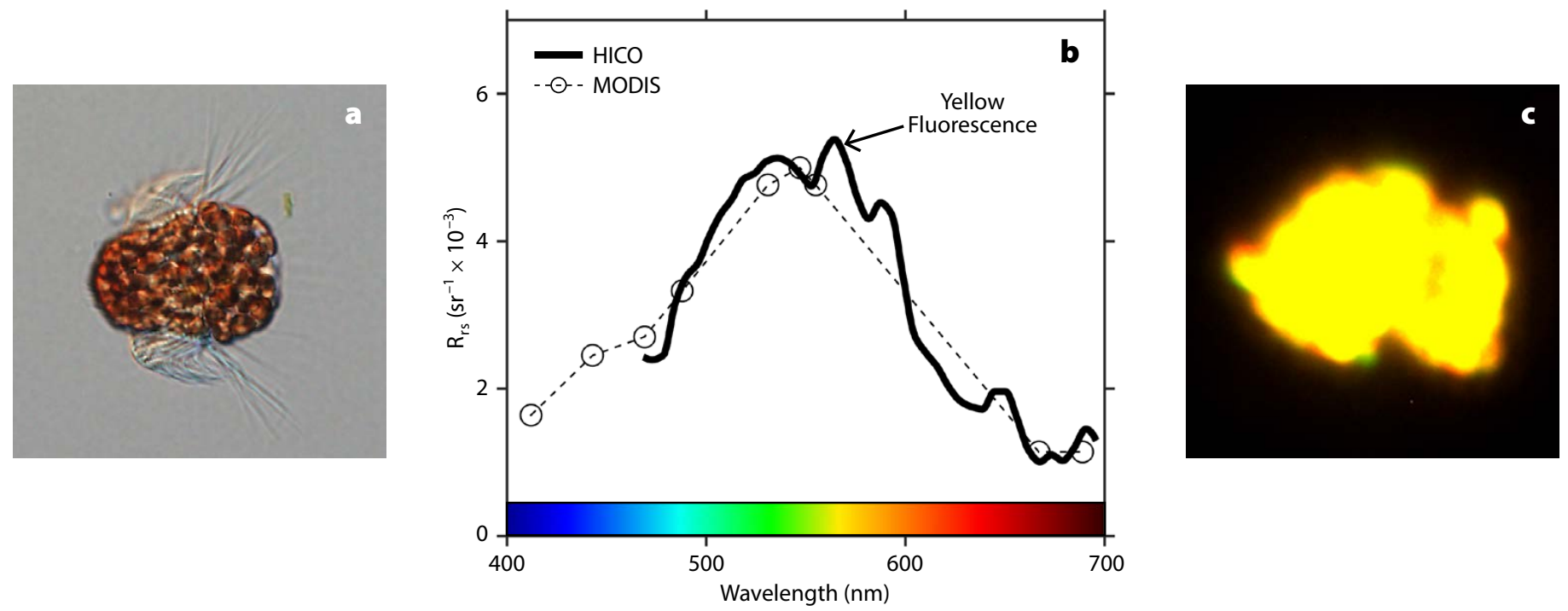

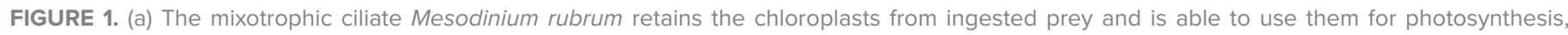

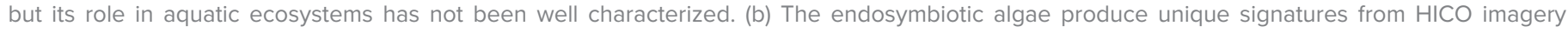

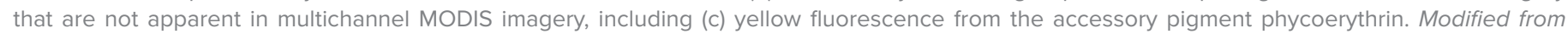
Dierssen et al. (2015) 
pal component analysis, derivative analysis, and other statistical methods. One suggestion was to produce products that represent the amount of absorption due to a given pigment (e.g., absorption peak heights) rather than pigment concentrations (Wang et al., 2016). An optical measure of pigment concentration may have less uncertainty and be more global in scope. Based on local knowledge, a data user could then characterize phytoplankton community composition based on pigment assemblages within a given region.

Moving forward, approaches that further incorporate the roles of scattering and fluorescence in differentiating phytoplankton may be fruitful (e.g., Figure 1). As shown in unpublished data at the workshop, other optical properties, such as absorption by nonalgal particles, may also provide some ability to differentiate certain PGs (Aimee Neeley, NASA, pers. comm., 2019; Alison Chase, University of Maine, pers. comm., 2019). The advances in polarimetry should also be considered in solving this problem. Finally, algorithms may also become more probabilistic and incorporate diverse streams of ancillary data (e.g., temperature, salin-

\begin{tabular}{|c|c|}
\hline Biogeochemical Modeling & $\begin{array}{l}\text { - Species composition } \\
\text { - Nutrient cycling } \\
\text { - Export of carbon, nitrogen, etc. }\end{array}$ \\
\hline Ecological Indicators & $\begin{array}{l}\text { - Hypoxia } \\
\text { - Eutrophication } \\
\text { - Informed monitoring and assessment }\end{array}$ \\
\hline Ecological Processes & $\begin{array}{l}\text { - Primary producers } \\
\text { - DMS producers } \\
\text { - Trophic dynamics and food web efficiency }\end{array}$ \\
\hline Global Change & $\begin{array}{l}\text { - Latitudinal distributional shifts } \\
\text { - Phenology shifts }\end{array}$ \\
\hline Fisheries & $\begin{array}{l}\text { - Finding fish } \\
\text { - Locations/monitoring for aquaculture } \\
\text { - Shellfish food safety }\end{array}$ \\
\hline $\begin{array}{c}\text { Harmful Algal Blooms (HABS) } \\
\text { and Human Health }\end{array}$ & $\begin{array}{l}\text { - Detecting types of blooms } \\
\text { - Finding probabilistic toxin production } \\
\text { - Forecasts and warnings to communities }\end{array}$ \\
\hline Environmental Reporting & $\begin{array}{l}\text { - Assessing compliance to thresholds } \\
\text { - Species identification } \\
\text { - Detecting anomalies }\end{array}$ \\
\hline
\end{tabular}

FIGURE 2. Potential applications for differentiating the fractional composition of various phytoplankton groups in aquatic ecosystems using hyperspectral imagery. DMS = Dimethyl sulfide. ity, daylength, wind speed, currents) to report probabilities of phytoplankton groups, such as harmful algal blooms, based on past data. For example, 13 years of phytoplankton species measurements in Belgian waters (Breton et al., 2006) showed that Phaoecystis globosa occurs every year in April/May but never from June to October. This information could be used to guide or quality control a PG algorithm.

\section{WHAT ARE THE APPLICATIONS FOR HYPERSPECTRAL ALGAL CHARACTERIZATION?}

Diverse applications exist for hyperspectral methods, ranging from ecological processes to human health aspects (Figure 2). As noted, the end users for such diverse applications include scientists, environmental managers, government agencies, private industry, and the general public. A follow-on study could better collate and incorporate the needs of end users and articulate what hyperspectral measurements coupled with other data and models can provide them. This would include the needs of coastal managers, biogeochemical modelers, aquaculture, and other end users.
- Species composition

- Hypoxia

- Informed monitoring and assessment

producers

-Trophic dynamics and food web efficiency

- Finding fish

or aquaculture

- Detecting types of bloom

Forecasts and warnings to communities

Assessing compliance to thresholds

- Species identification

Detecting anomalies 
types of measurements could be included in such a database, as long as the methods and potential uncertainty are identified. For example, skylight "contaminated" reflectance data are useful for skylight removal algorithm development and for algorithms that evaluate surface slicks, and skylight-impacted data can still be useful for approaches that use red/ near-infrared differences.

\section{Evaluate the Spectral Resolution}

Required for Phytoplankton Studies Coincident hyperspectral radiometry and IOPs with bandwidths $<5 \mathrm{~nm}$ are desirable for many inversion methods (Vandermeulen et al., 2017). Hyperspectral absorption measured with the filter pad method may provide better spectral resolution to differentiate pigments than in-water instrumentation with lower spectral resolution.

\section{Create Standardized} Metadata Protocol

Looking forward, it is extremely important for international research programs to follow standardized metadata protocols to ensure that the appropriate ancillary and methodological information are provided with each data set. Participants discussed lessons learned with past data compilation efforts, such as benthic reflectance measures. A future project goal is to work toward standardizing templates for metadata so that they work intelligently with radiometric, IOP, and phytoplankton composition data.

\section{Reanalyze Optical Terminology}

Some of the terms in use in the ocean optics community are not consistent with terminology in the wider field of environmental optics and do not accurately describe new advances made in our science. For example, terminology such as "nonalgal particles" and "colored dissolved organic matter" can be inaccurate and confusing to the broader audience. Even the terminology "ocean" optics is in question, because fundamentally similar satellite data and processing algo- rithms and validation requirements apply to aquatic ecosystems in general, including coastal, estuarine, and inland waters.

\section{Provide Guidance for}

Identifying Phytoplankton

\section{Groups Using High-Performance}

\section{Liquid Chromatography}

Various methods, such as CHEMTAX, are used to assess phytoplankton composition from pigment information. There seems to be a general lack of direction and training in robustly applying these methods and quantifying the uncertainty in retrievals. Some consistency and guidance in general applications of these methods would be warranted. One potential suggestion was to incorporate a Hyperspectral Phytoplankton Training Workshop and Exchange that would cou- ple PG and optical expertise and allow students and other professionals to bring data and work alongside an expert.

\section{HOW DO WE RAPIDLY DEVELOP A GLOBAL HYPERSPECTRAL DATABASE NEEDED FOR ALGORITHM DEVELOPMENT AND VALIDATION?}

The community needs a global database that combines hyperspectral optical measures of reflectance and absorption with phytoplankton composition in order to develop and test algorithms. Some potential suggestions follow.

\section{Provide Funds for Historic \\ Data Reanalysis}

Collating and reanalyzing data that have already been collected is a cost-effective

\section{Box 1. Satellite Sensors}

AVIRIS: Airborne Visible/Infrared Imaging Spectrometer developed by NASA's Jet Propulsion Laboratory

CHIME: Copernicus Hyperspectral Imaging Mission for the Environment

CHRIS: Compact High Resolution Imaging Spectrometer aboard the European Space Agency's PROBA-1 satellite

DESIS: DLR (German Aerospace Center) Earth Sensing Imaging Spectrometer, a hyperspectral sensor developed and operated collaboratively by the DLR and Teledyne Brown Engineering

EnMAP: Environmental Mapping and Analysis Program, a German hyperspectral satellite mission

HICO: Hyperspectral Imager for the Coastal Ocean, an imaging spectrometer that was housed on the International Space Station

MODIS: Moderate Resolution Imaging Spectroradiometer, a key instrument aboard NASA's Terra and Aqua satellites

PACE: NASA's Plankton, Aerosol, Cloud, ocean Ecosystem mission

PRISM: Picosatellite for Remote-sensing and Innovative Space Missions, a technology pathfinder mission of the Intelligent Space Systems Laboratory at the University of Tokyo, Japan

PRISMA: Hyperspectral Precursor and Application Mission, a medium-resolution hyperspectral imaging mission of the Italian Space Agency

SCIAMACHY: An ESA imaging spectrometer whose primary mission was to perform global measurements of trace gases in the troposphere and stratosphere

SBG: NASA's Surface Biology and Geology mission (formerly HyspIRI)

TROPOMI: TROPOspheric Monitoring Instrument onboard the ESA Copernicus Sentinel-5 Precursor satellite 
measure for providing diverse data across the globe. Current publicly available databases do not contain the types of data required for this research. For example, concentration data are not routinely incorporated in the Ocean Biogeographic Information System (OBIS), and no coincident hyperspectral optics are associated with the files. Researchers have volumes of historic data submitted to various databases, but often do not have the funds and time to rework and compile data into a merged format. Providing a funding pool for data reanalysis is recommended.

\section{Add Hyperspectral Optics to}

\section{Ongoing Coastal Observatories}

Many coastal programs have routine data collection activities that incorporate monitoring of phytoplankton communities and concentrations. Few of these programs collect coincident radiometric data. A white paper under development by the working group focuses on how to incorporate cost-effective hyperspectral radiometry and other optical measures into coastal programs.

\section{Target New Platforms}

Including hyperspectral optics and phytoplankton measurements into a variety of platforms, including ferries or other ships of opportunity, floats, moorings, and fixed platforms like AERONET-OC (the ocean color component of the Aerosol Robotic Network; Zibordi et al., 2009), aquaculture facilities, and new drone technology, may provide a wealth of data across diverse ecosystems. A future direction for the working group is to further report on new technologies and platforms for conducting coupled optical and phytoplankton measurements.

\section{Provide Shared Instrument}

Pools and Protocols

Following from past programs such as the NASA Sensor Intercomparison for Marine Biological and Interdisciplinary Ocean Studies (SIMBIOS) program, shared instrument pools and protocols may be useful for allowing researchers to collect data widely and in association with ships and field programs of opportunity. International data sharing policies would facilitate such activities.

\section{WHAT ARE THE BEST METHODS FOR VALIDATING PG PRODUCTS FROM SATELLITE MISSIONS?}

For validation of satellite data products, two steps can be distinguished. First, a comparison of in situ water reflectance measurements with near-simultaneous satellite measurement is needed to validate the atmospheric correction (including top-of-atmosphere calibration). Second, water product validation is needed, comparing satellite-derived measurements of PG products with corresponding inwater measurements. Steps for calibration and validation of radiometric data from satellite imagery have been outlined (CEOS, 2018; Ruddick et al., 2019). These studies show that long-term deployments (e.g., >1 year) of highly automated systems are needed to achieve a sufficient number of matchups for statistical analysis and meaningful identification of variability in atmospheric properties. However, the mooring locations identified for radiometric calibration may be quite different from those needed for phytoplankton studies.

The workshop identified as a key recommendation the co-location of longterm deployments of automated systems for measuring hyperspectral reflectance, inherent optical properties, and phytoplankton parameters (Lombard et al., 2019) across a diversity of water types, following successful programs like AERONET-OC (Zibordi et al., 2009). Such deployments are recommended pre-launch of satellite missions for development of algorithms. Field campaigns on ships are often undertaken to validate satellites and can provide a wealth of different types of in situ data but can be limited in terms of match-up data, biodiversity, and seasonality sampled. A more thorough treatise on best practices for satellite validation of phytoplankton products is warranted for both long-term deployments and short-term field campaigns to provide appropriate and consistent data for algorithm development and validation globally.

\section{HOW TO STRATEGIZE EFFORTS GLOBALLY?}

The next generation of hyperspectral ocean color satellites has been launched recently or is being developed for the next decade. As the International Ocean Colour Coordinating Group (IOCCG) website shows, the satellites span numerous countries and agencies, and have different specifications. Given the international nature of the missions, the final recommendation of the workshop is to develop international follow-on organizational task forces that would allow free and open data exchange and policy formulation. Potential organizations include:

- IOCCG Phytoplankton Group and

Hyperspectral Data Task Force

- Scientific Committee on Oceanic

Research (SCOR) Working Group on

International Data Sharing

- International Working Group on

Hyperspectral Airborne Missions

- International Partnerships with

Non-Governmental Organizations

The ultimate goal is to maximize the utility of hyperspectral imaging for assessing marine ecosystem biodiversity. Providing a more comprehensive understanding of marine biodiversity is critical for assessing responses to environmental change. @

\section{SUPPLEMENTARY MATERIALS}

The supplementary materials are available online at https://doi.org/10.5670/oceanog.2020.111.

\section{REFERENCES}

Bracher, A., M. Vountas, T. Dinter, J.P. Burrows, R. Röttgers, and I. Peeken. 2009. Quantitative observation of cyanobacteria and diatoms from space using PhytoDOAS on SCIAMACHY data. Biogeosciences 6(5):751-764, https://doi.org/ 10.5194/bg-6-751-2009.

Bracher, A., H.A. Bouman, R.J. Brewin, A. Bricaud, V. Brotas, A.M. Ciotti, L. Clementson, E. Devred, A. Di Cicco, S. Dutkiewicz, and others. 2017. Obtaining phytoplankton diversity from ocean color: A scientific roadmap for future development. Frontiers in Marine Science 4:55, https://doi.org/ 10.3389/fmars.2017.00055. 
